\title{
Therapeutic exercise attenuates neutrophilic lung injury and skeletal muscle wasting
}

\author{
D. Clark Files, ${ }^{1,2 *}$ Chun Liu, ${ }^{1}$ Andrea Pereyra, ${ }^{3,4}$ Zhong-Min Wang, ${ }^{3}$ Neil R. Aggarwal, ${ }^{5}$ \\ Franco R. D'Alessio, ${ }^{5}$ Brian T. Garibaldi, ${ }^{5}$ Jason R. Mock, ${ }^{5}$ Benjamin D. Singer, ${ }^{5}$ Xin Feng, ${ }^{6}$ \\ Raghunatha R. Yammani, ${ }^{7}$ Tan Zhang, ${ }^{3}$ Amy L. Lee, ${ }^{1}$ Sydney Philpott, ${ }^{1}$ Stephanie Lussier, ${ }^{1}$ \\ Lina Purcell, ${ }^{1}$ Jeff Chou, ${ }^{8}$ Michael Seeds, ${ }^{1,2}$ Landon S. King, ${ }^{5}$ Peter E. Morris, ${ }^{1,2}$ Osvaldo Delbono ${ }^{3}$
}

Early mobilization of critically ill patients with the acute respiratory distress syndrome (ARDS) has emerged as a therapeutic strategy that improves patient outcomes, such as the duration of mechanical ventilation and muscle strength. Despite the apparent efficacy of early mobility programs, their use in clinical practice is limited outside of specialized centers and clinical trials. To evaluate the mechanisms underlying mobility therapy, we exercised acute lung injury (ALI) mice for 2 days after the instillation of lipopolysaccharides into their lungs. We found that a short duration of moderate intensity exercise in ALI mice attenuated muscle ring finger 1 (MuRF1)-mediated atrophy of the limb and respiratory muscles and improved limb muscle force generation. Exercise also limited the influx of neutrophils into the alveolar space through modulation of a coordinated systemic neutrophil chemokine response. Granulocyte colony-stimulating factor (G-CSF) concentrations were systemically reduced by exercise in ALI mice, and in vivo blockade of the G-CSF receptor recapitulated the lung exercise phenotype in ALI mice. Additionally, plasma G-CSF concentrations in humans with acute respiratory failure (ARF) undergoing early mobility therapy showed greater decrements over time compared to control ARF patients. Together, these data provide a mechanism whereby early mobility therapy attenuates muscle wasting and limits ongoing alveolar neutrophilia through modulation of systemic neutrophil chemokines in lunginjured mice and humans.

\section{INTRODUCTION}

Early mobilization of critically ill patients has emerged as a promising therapy to improve outcomes of patients with the acute respiratory distress syndrome (ARDS) and other critical illnesses. Clinical research studies suggest that early mobilization of critically ill patients improves a number of meaningful variables, including intensive care unit length of stay (LOS), hospital LOS, delirium, hospital readmissions, and strength (1-5). The mechanisms underlying this therapy are currently unknown, although the improvements in a variety of organ systems (lung, muscle, and brain) suggest that early mobility/exercise may have widespread systemic effects. Despite the apparent efficacy of early mobility in critically ill patients, routine implementation of these programs outside the context of clinical trials remains low $(6,7)$. There are currently no pharmacologic therapies available to prevent or treat the extensive muscle wasting and functional disability present in survivors of critical illnesses, such as sepsis and ARDS (8). It is therefore important to understand the mechanisms underlying early mobility therapy so that targets can be identified and therapies can be developed to improve the outcomes of patients with these illnesses.

${ }^{1}$ Department of Internal Medicine-Pulmonary, Critical Care, Allergy and Immunologic Diseases, Wake Forest School of Medicine, Winston-Salem, NC 27157, USA. ${ }^{2}$ Wake Forest Critical IIIness, Injury and Recovery Research Center, Wake Forest School of Medicine, Winston-Salem, NC 27157, USA. ${ }^{3}$ Department of Internal Medicine-Gerontology, Wake Forest School of Medicine, Winston-Salem, NC 27157, USA. ${ }^{4}$ National Scientific and Technical Research Council (CONICET) and School of Medicine, National University of La Plata, 1900 La Plata, Argentina. ${ }^{5}$ Division of Pulmonary and Critical Care Medicine, Johns Hopkins Asthma and Allergy Center, Baltimore, MD 21205, USA. ${ }^{6}$ Department of Otolaryngology, Wake Forest School of Medicine, Winston-Salem, NC 27157, USA ${ }^{7}$ Department of Internal Medicine-Molecular Medicine, Wake Forest School of Medicine, Winston-Salem, NC 27157, USA. ${ }^{8}$ Division of Public Health Sciences, Wake Forest School of Medicine, Winston-Salem, NC 27157, USA

*Corresponding author. E-mail: dfiles@wakehealth.edu
We and others have shown that a mouse model of ARDS [referred to as acute lung injury (ALI) mice] develops profound skeletal muscle atrophy and weakness, which replicate many of the aspects of muscle wasting observed in patients with $\operatorname{ARDS}(1-5,9,10)$. Initiation of muscle atrophy in this model occurs temporally with lung injury, is associated with nuclear factor $\kappa \mathrm{B}(\mathrm{NF}-\kappa \mathrm{B})$ muscle activity, and depends on the expression of the muscle-specific E3 ubiquitin ligase muscle ring finger 1 (MuRF1) protein, which ubiquitinates contractile proteins for proteasomal-mediated degradation $(6,7,9,10)$. Although previous animal studies have shown that preconditioning mice with exercise can reduce the response to subsequent lung injury $(8,11)$, the ability of exercise to therapeutically improve outcomes after lung injury is unknown.

We exercised ALI mice after lung injury to evaluate the effects of therapeutic exercise on lung and muscle injury. Exercise led to marked improvements in lung, limb, and respiratory muscle injury. In this model, exercise altered expression of a systemic cytokine and chemokine response involving neutrophilic bone marrow mobilization and recruitment. Therapeutic exercise reduced neutrophil alveolitis, MuRF1mediated skeletal muscle atrophy, and muscle weakness. Therapeutic blockade of granulocyte colony-stimulating factor (G-CSF), a known biomarker of poor outcomes in patients with ARDS (12), recapitulated some features of the exercise phenotype in ALI mice. In addition, human patients with acute respiratory failure (ARF) undergoing early mobility therapy showed greater decrements in G-CSF levels over time, compared to non-exercised patients, confirming the relevance of G-CSF as a mediator of the improved outcomes of mobilized/exercised critically ill patients. These data in mice and humans suggest a unifying mechanism underlying the beneficial effects of therapeutic exercise in mice and humans with lung injury. 


\section{RESULTS}

\section{ALI mice exhibit marked lung inflammation}

Instillation of lipopolysaccharides (LPS) into the lungs of 2-month-old wild-type (ALI) mice resulted in acute lung inflammation, marked by predominant alveolar and interstitial cellular infiltration and interstitial thickening at day 3 , which was quantified by digital morphometry software (Fig. 1, A and B). Day 3 bronchoalveolar lavage (BAL) cells and protein (Fig. 1C) were also similarly increased in ALI mice at this time point of maximal lung injury, on the basis of our previous work with this model (10).

\section{ALI mice have reduced physical activity}

We have previously shown that ALI mice display profound limb muscle wasting, mediated in part by the muscle expression of MuRF1 protein, which is up-regulated in the limb muscles of ALI mice during days 1 to 4 after LPS instillation (10). Here, we observed that ALI mice exhibited reduced physical activity, quantified by both traveling (Fig. 2A) and rearing activity (Fig. 2B).

To determine whether muscle wasting in ALI mice phenotypically differs from that induced by reduced activity or immobility, we adopted
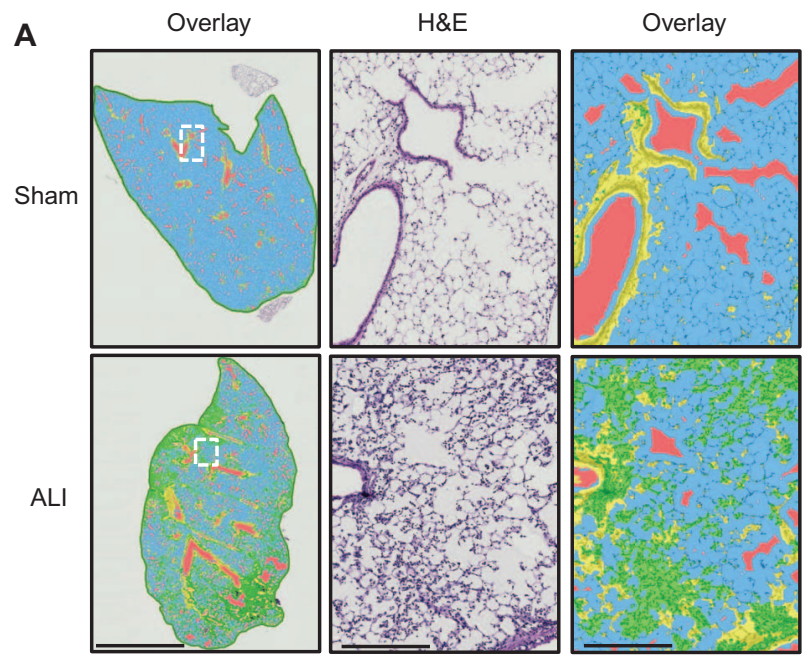

B
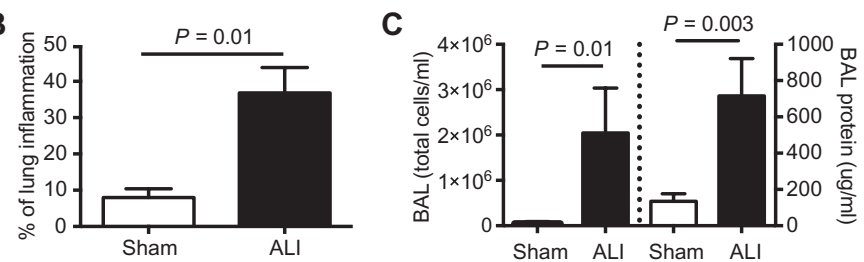

Fig. 1. Intratracheal instillation of LPS causes lung injury in mice. (A) Representative hematoxylin and eosin (H\&E)-stained left lung sections of sham and ALI mice are shown and were evaluated for parenchymal inflammation using digital imaging software at low (left panels) or high (middle and right panels) magnification at day 3. In overlay images, normal alveolar space (blue), airways (red), bronchial epithelium (yellow), and injured areas (green) can be identified. Scale bar, $3 \mathrm{~mm}$ (left panels); $300 \mu \mathrm{m}$ (middle and right panels). (B) Quantification of the percentage of lung inflammation based on digital morphometry images. (C) Bronchoalveolar total cell counts (left axis) and protein levels (right axis) in sham and ALI mice on day 3. $n=$ 4 to 6 per group. Data were analyzed using the Student's two-tailed $t$ test. a model of hindlimb immobilization in sham and ALI mice. As previously reported, one hindlimb is secured in a flexed position so that the muscles of that limb are completely immobile (13). The contralateral rear hindlimb serves as an internal control. We applied this hindlimb immobilization model to sham and ALI mice at day 0 and harvested the mice at day 3 . We found that complete limb immobility increased MuRF1 mRNA (Fig. 2C) and protein levels (Fig. 2, D and E) in the tibialis anterior muscle in sham mice as previously reported (13). However, ALI control conditions were associated with greater MuRF1 levels compared to sham immobilized conditions. ALI + immobilized muscles failed to display further significant MuRF1 up-regulation, showing that most MuRF1 activation in ALI mice can be accounted for by ALI conditions alone and that muscle wasting in ALI mice is phenotypically different from that induced by immobility. We hypothesized that this reduction in physical activity was maladaptive and sought to determine whether increased activity/exercise after instillation of LPS would attenuate the muscle wasting that occurs in ALI mice.

\section{Therapeutic exercise attenuates skeletal muscle atrophy in ALI mice}

We designed exercise regimens that would be expected to approximate 40 to $60 \%$ maximal oxygen consumption $\left(\mathrm{VO}_{2} \max \right)$ in a healthy young C57BL/6J mouse (14), ranging from 5 min daily to $35 \mathrm{~min}$ twice daily starting 24 hours after the instillation of LPS (day 1). We found that exercise regimens ranging from 5 min daily to 25 min twice daily (table S1) attenuated the loss of muscle mass in ALI mice in individual muscles with a variety of fiber-type compositions. Tibialis anterior (type IIa/x and type IIb fibers), extensor digitorum longus (type Ilax and type IIb fibers), and soleus (type IIa/x, type IIb, and type I fibers) muscle mass loss was attenuated at most doses of exercise. At the highest intensity and duration of exercise, $35 \mathrm{~min}$ twice daily, exercise failed to attenuate the loss of muscle mass in ALI mice (fig. S1A). Figure $2 \mathrm{~F}$ demonstrates the ALI and exercise regimen used in the remaining experiments in this article at a dose of 25 min twice daily, hereby referred to ALI + Ex mice. We found a trend toward improved survival in ALI + Ex mice using this dose of exercise, but the difference did not reach statistical significance $(97 \%$ ALI + Ex versus $90 \%$ ALI, $P=0.06$ ) (fig. S1B).

We further examined the myofiber composition of the soleus and diaphragm in sham, ALI, and ALI + Ex mice. Similar to our previous reports, we found that ALI conditions caused type II myofiber atrophy in the limb muscle (soleus), with relative preservation of type I myofibers. In contrast, ALI mice displayed atrophy of both type I and type II myofibers of the diaphragm. Exercise in ALI mice attenuated type II myofiber atrophy in both the soleus and the diaphragm (Fig. 3, A to C). These data show that therapeutic exercise in ALI mice attenuates type II myofiber atrophy.

\section{Therapeutic exercise in ALI mice attenuates MuRF1-mediated atrophy and improves muscle performance}

We have previously shown that up-regulation of MuRF1 protein is necessary for the skeletal muscle atrophy that occurs in this model at days 3 to $4(10,15)$. Here, we confirmed that ALI mice had increased MuRF1 mRNA and protein expression compared to sham mice in all muscles evaluated. Notably, ALI + Ex mice exhibited reduced MuRF1 mRNA and protein expression in both the limb muscles and the diaphragm compared to ALI mice (Fig. 3, D to F). 

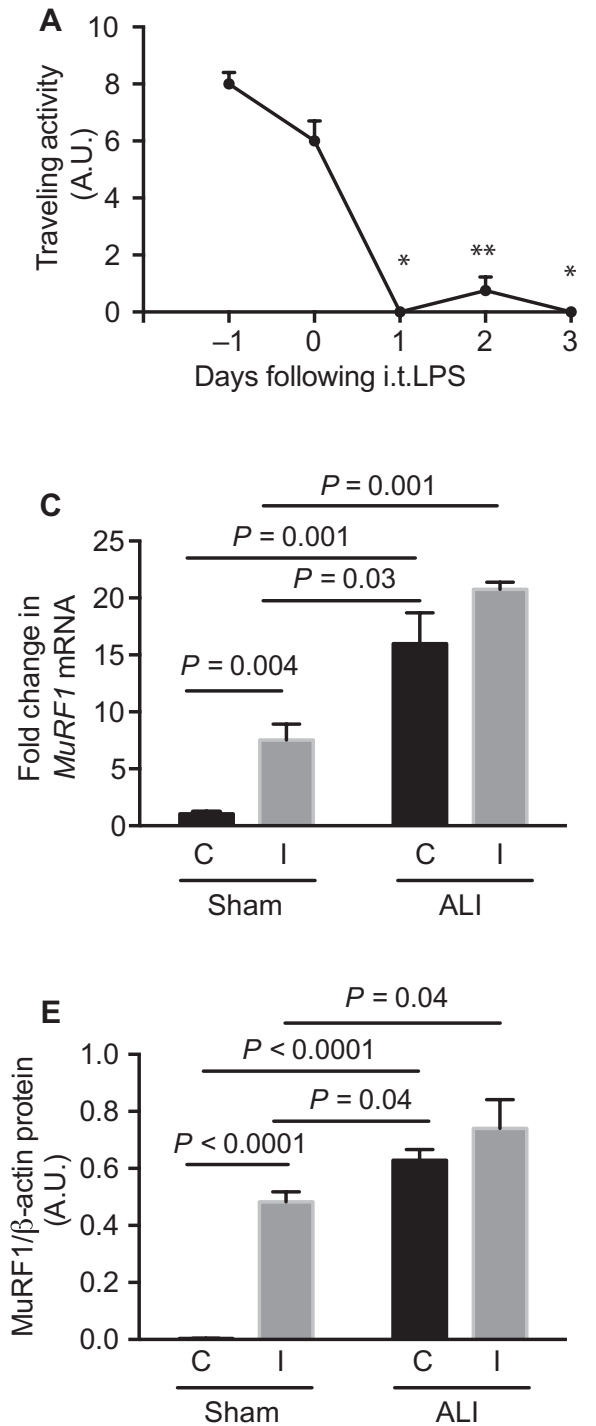

Fig. 2. Physical activity is reduced in ALI mice. (A and B) Traveling (A) and rearing (B) activity was measured 2 days before and 3 days after instillation of intratracheal LPS (i.t.LPS). A.U., arbitrary unit. (C to E) Sham and ALI mice underwent hindlimb immobilization at day 0 and were harvested at day 3 . MURF1 mRNA (C) and protein (D and E) levels were quantified in the tibialis anterior muscle of sham and ALI control (C) and immobilized (I) muscles. (F) Experimental scheme of most exercise experiments detailed within this article. Deviations from this scheme are noted within the text. Values represent means \pm SEM. $n=4$ per group (A and $\mathrm{B}$ ); $n=3$ to 4 per group (C to E). (A and $\mathrm{B}){ }^{*} P=0.00002,{ }^{* *} P=0.0003$, and ${ }^{* * *} P=$ 0.00006 with Bonferroni correction at all time points compared to time 0 using the Student's two-tailed $t$ test.

To determine whether therapeutic exercise alters muscle MuRF1 expression in the absence of ALI, we exercised sham mice (Sham + Ex) with the same exercise protocol used in the ALI + Ex mice. Exercise alone had no effect on MuRF1 mRNA in the absence of ALI (Fig. 3D).

We and others have shown that ALI mice have increased muscle NF- $\kappa \mathrm{B}$ activation $(9,10)$, which is a known upstream mediator of MuRF1 induction (16). Here, we found that ALI + Ex mice had reduced muscle NF- $\kappa \mathrm{B}$ activation compared to ALI mice at day 2, measured by reduced phosphorylation of the NF- $\mathrm{B}$ subunit $\mathrm{p} 65$ in nuclear preparations (Fig. 3, G and $\mathrm{H}$ ).
We have previously shown that ALI mice develop limb muscle weakness, with a $~ 30 \%$ decrease in maximal tetanic force compared to sham mice (10). To determine whether exercise in ALI mice preserves muscle function, we isolated the soleus muscle and stimulated the muscle directly via electrical field stimulation according to previously published methods (17) adapted for the soleus muscle. We found a 20 and $14 \%$ increase in twitch absolute and specific forces, respectively, in ALI + Ex compared to ALI mice (Fig. 3, I and J). Likewise, absolute and specific tetanic forces were increased at all measured frequencies in ALI + Ex mice. Maximal tetanic absolute and specific forces were increased 16 and $10 \%$, respectively, in ALI + Ex versus ALI mice (Fig. 3, K and $\mathrm{L}$ ). We found no difference in soleus muscle fatigability between ALI versus ALI + Ex mice (fig. S1C). These data demonstrate that exercise in ALI mice reduces MuRF1-mediated atrophy and improves muscle function in ALI mice.

\section{Exercise in ALI mice attenuates neutrophil alveolitis}

Because muscle atrophy and function were improved in ALI + Ex mice, and muscle atrophy is temporally correlated with lung injury in this model (10), we quantified lung injury parameters in the three groups. Surprisingly, we found that exercise in ALI mice limited the influx of neutrophils into the alveolar compartment. BAL total cells were reduced at day 3 in ALI + Ex compared to ALI mice, with reductions in monocytes/macrophages, neutrophils, and lymphocytes at this time point of peak lung inflammation (Fig. 4, $\mathrm{A}$ and $\mathrm{B})$. We also found reduced total cell counts in the alveolar space of ALI + Ex mice at most doses of exercise studied (fig. S1D). Neutrophils are the predominant inflammatory cell in the lung at day 3 (Fig. 4B), so the reduction in alveolar cell counts with exercise can primarily be accounted for by reduced alveolar neutrophilia. To evaluate the possibility that exercise increased alveolar neutrophil apoptosis, we labeled BAL neutrophils for the apoptosis markers 7-aminoactinomycin D and annexin $\mathrm{V}$. We found no evidence of increased neutrophil apoptosis in the alveolar space of ALI + Ex mice (fig. S1E). In contrast to the reduced cells found in the alveolar compartment, cell-free BAL total protein or albumin was not reduced in ALI + Ex mice (fig. S1, F and G). These data suggest that therapeutic exercise in ALI mice reduces neutrophil alveolitis, a finding associated with poor outcomes in $\operatorname{ARDS}(18,19)$. 

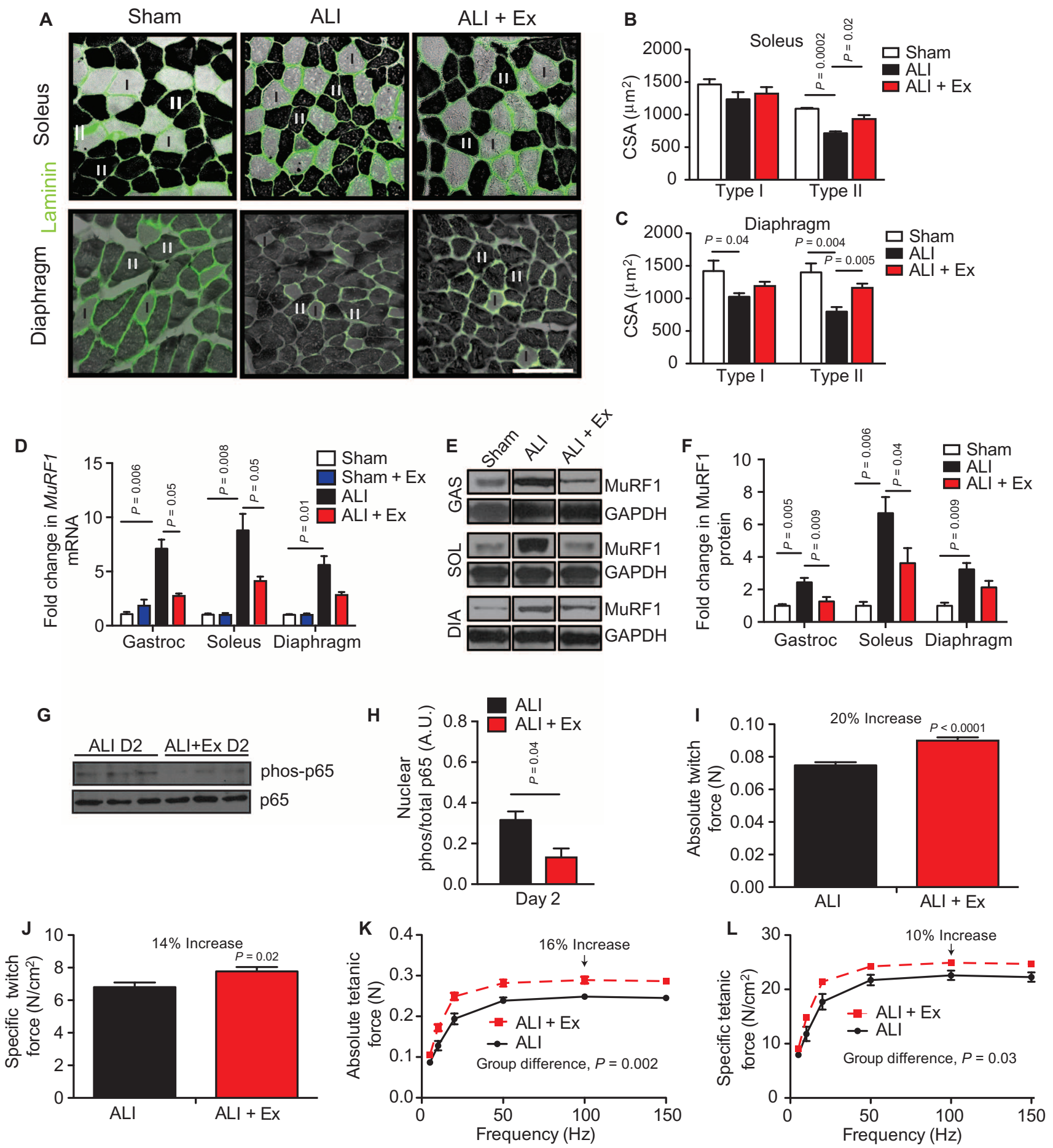

Fig. 3. Therapeutic exercise attenuates ALI-induced muscle atrophy and improves muscle performance. (A) Type I (light) and II (dark) myofibers of the soleus and the diaphragm were identified by adenosine triphosphatase (ATPase) and laminin (green) staining. Scale bar, $100 \mu \mathrm{m}$. (B and C) Muscle fiber cross-sectional area (CSA) was quantified in sham, ALI, and ALI + Ex mice. (D to F) MuRF1 mRNA (D) and protein ( $E$ and F) levels normalized to GAPDH (glyceraldehyde-3-phosphate dehydrogenase) in gastrocnemius (GAS), diaphragm (DIA), and soleus (SOL) muscles. (G and $\mathbf{H}$ ) Phosphorylated

and total p65 protein from gastrocnemius nuclear extracts. D2 (day 2). (I to L) Ex vivo isolated soleus ( $\mathrm{I}$ and $\mathrm{J}$ ) absolute and specific twitch and ( $\mathrm{K}$ and $\mathrm{L}$ ) tetanic contractile force measurements. Values represent means \pm SEM. All experimental time points are at day 3 , other than $(\mathrm{G})$ and $(\mathrm{H})$, which are at day 2. $n=4$ to 5 per group (A to $\mathrm{D}$ ); $n=6$ to 8 per group of two combined experiments $(\mathrm{F}) ; n=3$ per group $(\mathrm{G}$ and $\mathrm{H}) ; n=4$ animals and 8 muscles per group (I to L). Data were analyzed using the Student's two-tailed $t$ test or analysis of variance (ANOVA) for group differences with multiple time points. 
A

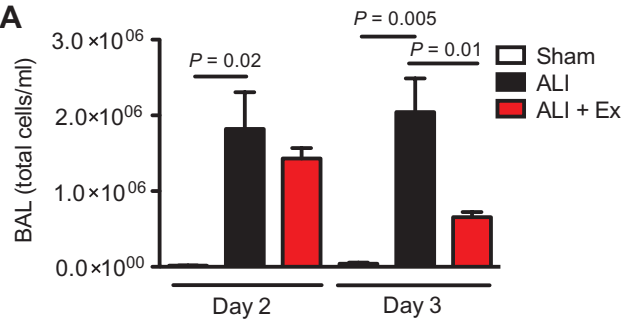

C

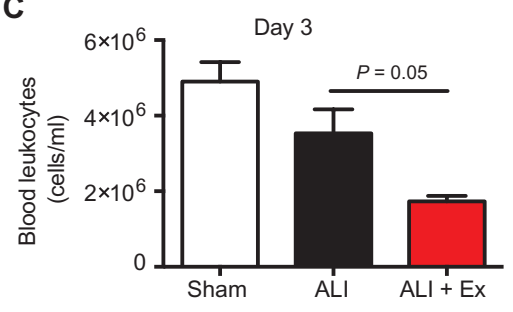

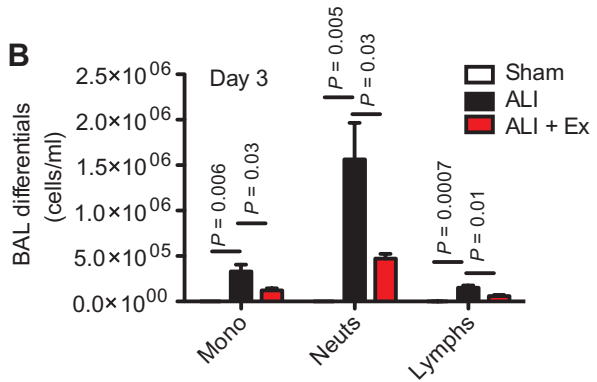

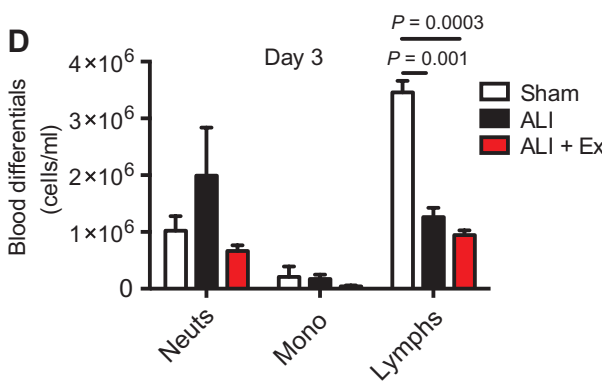

Fig. 4. Therapeutic exercise reduces alveolar neutrophilia. (A and $B$ ) $B A L$ total leukocyte $(A)$ and differential cell counts (B) in sham, ALI, and ALI + Ex mice at specified time points. (C and D) Total blood leukocytes (C) and differential cell counts (D) were measured in sham, ALI, and ALI + Ex mice at day 3. $n=3$ to 6 per group. Data were analyzed using the Student's two-tailed $t$ test.

\section{Exercise in ALI mice alters the systemic neutrophil cytokine/chemokine response}

We found that exercise (ALI + Ex) reduced total blood leukocytes compared to ALI mice without exercise (Fig. 4C). ALI conditions shifted blood leukocytes from a predominant lymphocytic (sham mice) to a predominant neutrophilic (ALI mice) profile (Fig. 4D). The reduction in total leukocytes in ALI + Ex mice compared to ALI mice was primarily accounted for by an about twofold reduction in blood neutrophils (Fig. 4D).

To evaluate for potential systemic mediators underlying the improvements in lung and muscle injury seen in ALI + Ex mice, we performed an unbiased inflammatory biomarker array on 144 murine cytokines in ALI and ALI + Ex mice. Table 1 lists the top up- and down-regulated proteins with $P \leq 0.08$ in the array. We found that 10 proteins were significantly differentially regulated $(P \leq 0.05)$ in ALI + Ex versus ALI mice (Table 1). Seven of the 10 significantly differentially regulated proteins $(P \leq 0.05$; starred and bolded in Table 1$)$ and 9 of the 15 potentially differentially regulated proteins $(P \leq 0.08$; starred in Table 1) have known roles in neutrophil mobilization and recruitment. Data from all 144 proteins can be found in table S2. Notably, therapeutic exercise did not change the levels of tumor necrosis factor- $\alpha$ (TNF- $\alpha$ ), interleukin-6 (IL-6), or IL-10 (table S2), which have been implicated in the protective effect of 5 weeks of prophylactic aerobic exercise in lung-injured mice (11). These data suggest that therapeutic exercise in ALI mice has specific immunomodulatory effects, most notably on regulation of cytokines and chemokines involved in neutrophil mobilization and migration. This effect appears mechanistically different from chronic aerobic "preconditioning" exercise.

Exercise reduces systemic G-CSF concentrations in ALI mice We chose to focus further investigation on the top down-regulated cytokine in our array, G-CSF, because of its well-described mechanisms of action (20) and its known relevance in human ARDS $(12,19)$. G-CSF protein was markedly up-regulated ( 100 -fold) in the plasma of ALI compared to sham mice. Exercise profoundly reduced systemic G-CSF levels in ALI mice (Fig. 5A). We also quantified IL-17A, IL-17F, and IL-23, known upstream regulators of G-CSF production. We found similar trends in the pattern of expression of these cytokines, namely, reduction in systemic levels with exercise, although only IL-17F reached statistical significance (Fig. 5, B to D).

\section{Blockade of the G-CSF receptor in vivo reduces neutrophilic alveolar influx but does not prevent muscle atrophy}

To determine the specificity of the role of G-CSF in mediating neutrophilic lung injury and muscle wasting, we systemically administered G-CSF receptor-blocking antibodies to ALI mice (ALI + G-CSFR Ab), starting 24 hours after i.t.LPS, and harvested the mice at day 3 . We found that G-CSFR blockade reduced cells in the alveolar space compared to ALI mice receiving an isotype control antibody (ALI + isotype) to a similar degree to that seen in ALI + Ex mice (Fig. 5E). In contrast, blockade of the G-CSFR did not attenuate soleus type II myofiber atrophy as seen in the ALI + Ex mice (Fig. 5, F and G). Likewise, MuRF1 protein levels in ALI + G-CSFR Ab-treated mice were not reduced compared to those in ALI + isotype mice (Fig. 5, $\mathrm{H}$ and I) as they were in ALI + Ex mice. We also administered G-CSFR Ab starting at day -1 or 0 and continued daily through day 2 until harvest at day 3. Similarly, we found that G-CSFR blockade reduced cellular alveolitis in ALI mice (fig. S2A) but did not attenuate MuRF1-mediated atrophy (fig. S2, B to D).

These data suggest that G-CSF, potentially driven by IL-17F and/or IL-23, is a key regulator of the improved alveolar neutrophilic injury in exercised ALI mice, although the attenuation in MuRF1-mediated muscle atrophy in ALI + Ex mice occurs through a G-CSF-independent mechanism.

\section{Therapeutic exercise in humans with ARF is associated with reduced plasma G-CSF concentrations over time}

To confirm the relevance of our murine studies, we obtained banked plasma from a randomized controlled pilot study of early mobilization (exercise) versus usual care (no exercise) in patients with ARF in the Wake Forest Medical Intensive Care Unit. The details of this study can be found in Supplementary Materials and Methods. Briefly, all patients randomized to exercise received passive range of motion of the extremities three times daily, replaced by active physical therapy as patient interaction improved, which included sitting on the edge of bed, standing, and walking based on a previously described protocol (1). The baseline characteristics of patients at enrollment in each arm can be found in table S3.

Respiratory failure patients randomized to exercise had a $68 \%$ reduction in G-CSF levels from baseline to day 7 versus a $29 \%$ reduction 
Table 1. Exercise in ALI mice induces a systemic neutrophil cytokine/ chemokine response. An unbiased inflammatory biomarker array of 144 proteins was performed on the plasma of ALI + Ex and ALI mice at day 3. $n=4$ per group. Displayed are the proteins with $P$ values $\leq 0.08$ in the ALI + Ex versus ALI mice. Proteins in bold have $P$ values $\leq 0.05$. All proteins can be found in table S2. Starred cytokines $\left(^{*}\right)$ have been previously implicated in neutrophil mobilization, activation, and/or migration.

\begin{tabular}{|c|c|c|c|c|}
\hline Response to exercise & Protein & Fold change & $\%$ Change & $P$ \\
\hline \multirow[t]{12}{*}{ Down-regulation } & G-CSF* & -2.6 & -160 & 0.02 \\
\hline & CXCL15* & -2.4 & -140 & 0.02 \\
\hline & $\mathrm{KC} C^{*}$ & -2.0 & -100 & 0.08 \\
\hline & Osteoprotegerin & -1.9 & -90 & 0.03 \\
\hline & GAS 6* & -1.7 & -70 & 0.04 \\
\hline & IL-17F* & -1.6 & -60 & 0.05 \\
\hline & VEGFR3 & -1.4 & -40 & 0.02 \\
\hline & IL-17B* & -1.2 & -20 & 0.02 \\
\hline & DKK-1 & -1.2 & -20 & 0.06 \\
\hline & IL-1 ra & -1.5 & -50 & 0.06 \\
\hline & CXCL10* & -1.5 & -50 & 0.06 \\
\hline & HGF & -1.3 & -30 & 0.07 \\
\hline \multirow[t]{3}{*}{ Up-regulation } & Galectin-1* & 2.1 & +110 & 0.009 \\
\hline & MMP-3 & 2.0 & +100 & 0.03 \\
\hline & MIP-2* & 1.3 & +30 & 0.02 \\
\hline
\end{tabular}

in control patients over this time interval (Fig. 6A). Despite randomization, baseline (day 1) levels of G-CSF were higher in the exercise group. We therefore normalized the data to adjust to the mean baseline level of G-CSF for each group (Fig. 6B). After this adjustment, we found that G-CSF was significantly reduced at the day $5(P=0.0538)$ and day $7(P=0.0072)$ time points in the exercise versus no exercise groups. These data add human relevance to the murine findings described above, which show that therapeutic exercise reduces systemic levels of G-CSF in ALI mice.

\section{DISCUSSION}

In these studies, we have identified mechanisms underlying the benefits of early mobility therapy demonstrated by previous clinical investigations $(1,2,4,5)$. We find that therapeutically treating ALI mice with moderate intensity exercise of 2 days' duration improved both skeletal muscle wasting and neutrophilic alveolar influx. Exercise appears to have specific immunomodulatory effects, most notably on limiting the mobilization and recruitment of neutrophils from the bone marrow to the lung by neutrophil cytokines and chemokines. G-CSF appears to be a key mediator of this process and is reduced in humans and mice with lung injury undergoing therapeutic exercise. The attenuation in muscle atrophy with exercise in ALI mice occurs independently of G-CSF, because inhibition of the G-CSFR did not improve muscle atrophy in ALI mice.

Our data support the concept that although neutrophils are critical for the early response to inflammation or infection in the lung, ongoing lung neutrophilia promotes lung injury (21). In mouse models of lung injury induced by endotoxin (22) or endotoxin plus oxygen (23), neutrophil depletion is protective. In addition, prolonged alveolar neutrophilia is associated with mortality in patients with $\operatorname{ARDS}(18,19,24)$. Our data suggest that therapeutic exercise in mice and humans may act as a rheostat to dampen ongoing neutrophil numbers by reducing G-CSF, which may, in turn, be driven by down-regulation of IL-17F and IL-23 (25). G-CSF's activity in this model is likely occurring through binding to the G-CSFR, which is present on neutrophils, monocytes, and bone marrow hematopoietic stem/progenitor cells (26), as well as myoblasts (27) and endothelial cells (28).

The administration of recombinant G-CSF ( $\mathrm{rG}-\mathrm{CSF}$ ) can cause lung injury in rats $(29,30)$ and has been associated with ARDS in immunocompromised patients $(31,32)$, although systemic administration of rG-CSF in nonimmunocompromised patients with pneumonia does not appear to lead to an increase in ARDS (33-35). Suratt et al. demonstrated in a large cohort of ARDS patients that endogenous G-CSF levels are positively correlated with duration of mechanical ventilation, organ failure, and mortality (12). Our results in mice suggest that the endogenous G-CSF contributes to alveolar neutrophilic injury and that therapeutic exercise helps reduce G-CSF over time. Collectively, these data suggest that G-CSF is at least a biomarker and may be pathogenic in ARDS. In contrast to this, the lack of ARDS development with rG-CSF administration in pneumonia patients suggests that rG-CSF alone is not sufficient to cause/promote ARDS.

The actions of G-CSF in skeletal muscle have also previously been examined. Hara et al. have shown that the G-CSFR is transiently expressed in cardiotoxin-injured regenerating mouse skeletal muscle: blockade of the G-CSFR is deleterious for muscle regeneration in this model, suggesting that G-CSF is beneficial for repair after muscle injury (27). Although we found no harm to the skeletal muscle after blocking the G-CSFR in our model, this issue and its potential negative implications for muscle regeneration after injury will need to be considered.

In addition to the well-described role of G-CSF in neutrophil mobilization and activation (36), 9 of the 15 proteins in our array with $P<$ 0.08 , including CXCL15 (lungkine) (37), keratinocyte chemoattractant (KC) (23), growth arrest specific 6 (GAS 6) (38), IL-17F/B (39), CXCL10 (40), galectin-1 (41), and macrophage inflammatory protein-2 (MIP-2) (23), have been implicated in neutrophil migration. Because these chemokines alter neutrophil migration, our data suggest that exercise induces a coordinated response that reprograms bone marrow mobilization, activation, and migration of neutrophils. Further studies are needed to define the roles of each of these cytokines/chemokines in this model, as well as the other proteins that are differentially regulated in ALI + Ex mice.

Previous work has demonstrated that 4 to 8 weeks of prophylactic exercise reduce lung injury in mouse models of asthma $(42,43)$ or ALI (11). Gonçalves et al. showed that 4 to 6 weeks of prophylactic aerobic exercise reduced lung neutrophilia and TNF- $\alpha$ and increased IL- 6 and IL-10 after LPS injury (11). We found no change in TNF- $\alpha$, IL-6, or IL-10 with 2 days of therapeutic exercise after lung injury. This suggests that preconditioning and therapeutic exercise both benefit the lung response to LPS-induced injury, although the mechanisms mediating these benefits may differ.

Our study also has implications for the timing and delivery of early mobility therapies in humans with ARDS. Because exercise in our lunginjured mice affected cytokines involved in neutrophil mobilization 

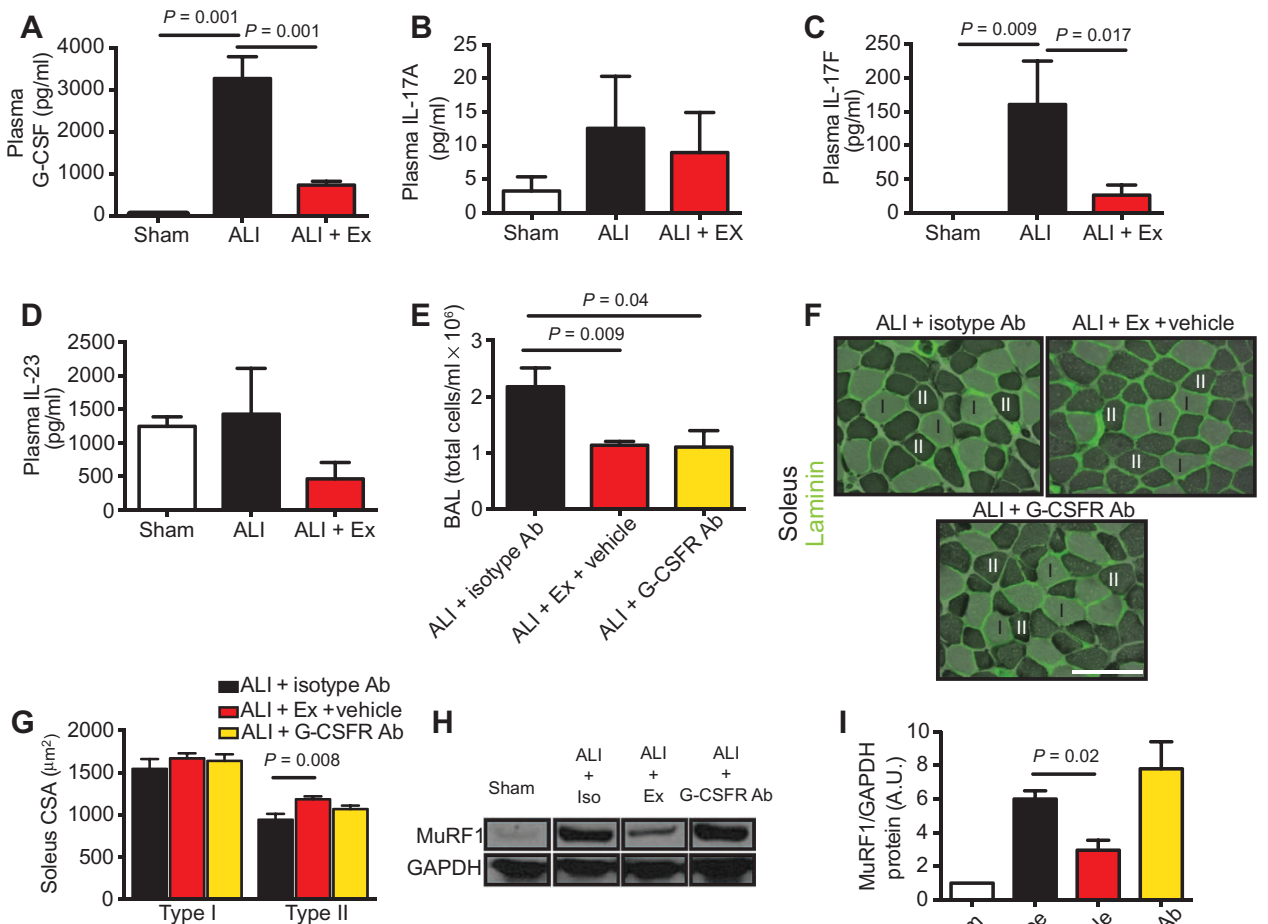

Fig. 5. Blockade of G-CSF activity limits lung injury but does not attenuate muscle atrophy. (A to D) G-CSF, IL-17A, IL-17F, and IL-23 protein quantification in the plasma of sham, ALI, and ALI + Ex mice. (E) BAL cell counts were quantified at day 3 after systemic administration of isotype antibody (ALI + isotype $A b$ ), exercise (ALI + Ex + vehicle), or G-CSFR-blocking antibody (ALI + G-CSFR Ab) 1 day after i.t.LPS administration. (F) Type I (light) and II (dark) myofibers of the soleus were identified by ATPase and laminin (green) staining. (G) Cross-sectional area was quantified in ALI + isotype Ab, ALI + Ex + vehicle, and $\mathrm{ALI}+\mathrm{G}$-CSFR Ab mice. Scale bar, $100 \mu \mathrm{m}$. (H and I) Soleus muscle lysates were probed for MuRF1 protein and normalized to GAPDH in sham, ALI + isotype Ab, ALI + Ex + vehicle, and ALI + G-CSFR Ab mice (H) and quantified by densitometry (I). $n=3$ to 7 per group. Data were analyzed using the Student's two-tailed $t$ test.

not attenuate muscle wasting. Intense exercise in physically fit humans has been associated with leukopenia and an increased risk of upper respiratory tract infections after training (44). Exercise doses ranging from 5 to 25 min twice daily generally attenuated the loss of muscle mass in ALI mice; conversely, the highest exercise dose $35 \mathrm{~min}$ twice daily either increased or failed to attenuate (depending on the muscle studied) muscle wasting. This response to exercise, in which low to moderate exercise is protective but the highest intensity exercise is detrimental, fits with the previously proposed "J-curve" immune response to exercise observed in humans (45). Future clinical studies should focus on exercise intensity dosing strategies in ARDS patients.

We have previously shown that muscle wasting in ALI mice is associated with increased activity of muscle NF- $\kappa \mathrm{B}(10)$, a known promoter of muscle atrophy and upstream mediator of MuRF1 expression (16). Others have shown that exercise can reduce NF- $\kappa \mathrm{B}$ lung activation in injured mouse lungs (42), and we also find that therapeutic exercise acutely reduces muscle $\mathrm{NF}-\kappa \mathrm{B}$ activation, which may drive the down-regulation of MuRF1 expression in $\mathrm{ALI}+\mathrm{Ex}$ mice. Previous investigators have shown that the NF- $\kappa \mathrm{B}$-mediated effect of exercise occurs through a glucocorticoid receptor-dependent mechanism (46).

Last, and in line with our previous studies, ALI mice developed type II myo-

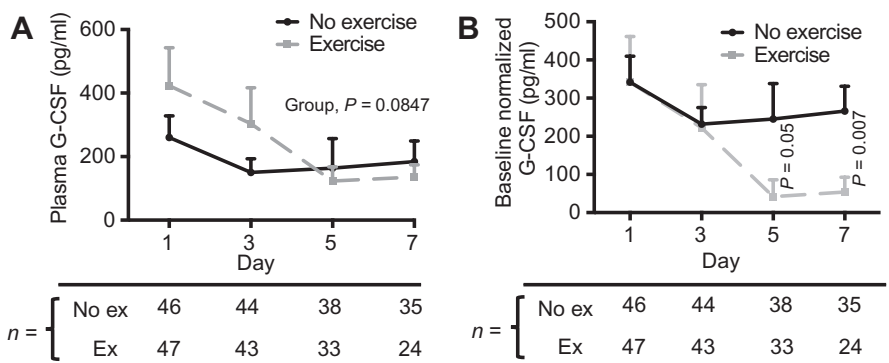

Fig. 6. Early mobility therapy reduces plasma G-CSF over time in patients with ARF. (A) G-CSF concentrations measured by enzyme-linked immunosorbent assay (ELISA) at specified time points on plasma samples from patients in a randomized controlled study of early mobility therapy. (B) G-CSF concentrations normalized to the mean baseline concentration of G-CSF of each group at specific time points. Group differences over time were analyzed using the generalized estimating equation (GEE) method, and time point differences in (B) were compared using the Mann-Whitney test. ex, exercise.

and migration, and neutrophil influx into the alveolar space is an early event during the time course of ARDS, our findings imply that early mobility therapies in the critically ill should start as early as possible. We also found that the highest intensity of exercise in ALI mice did fiber atrophy after lung injury. We found both type I and type II myofibers in the diaphragm atrophy in ALI mice, consistent with a recent report of diaphragmatic biopsies in critically ill humans (47). Therapeutic exercise in ALI mice attenuated type II myofiber atrophy in the limb muscle and diaphragm but did not improve type I myofiber atrophy in the diaphragm. Because MuRF1 is preferentially expressed in type II myofibers (48), we hypothesize that this is the reason for the discrepancy, in that exercise reduces whole-muscle MuRF1 expression in both the limb and the diaphragm but does not attenuate the type I myofiber atrophy in the diaphragm. It is also noteworthy that exercise also had beneficial effects on limb muscle function that were independent of muscle atrophy, given that most improvement in force production in the soleus was from specific, not absolute, force in ALI + Ex mice.

There are limitations to our study. We have not examined neutrophil function in ALI + Ex mice. The coordinated neutrophil cytokine modulation seen with exercise would suggest changes in neutrophil functionality, including possibly their ability to migrate across endothelial/ epithelial barriers. The roles of other differentially regulated cytokines besides G-CSF in ALI + Ex mice were not examined in this study. Future work should examine the role of therapeutic exercise in mice with sepsis, such as the cecal ligation and puncture model. Additionally, the G-CSF plasma measurements in mobilized human ARF 
patients need to be confirmed in a larger cohort of patients. Future studies are needed to clarify these issues, as well as the mechanism responsible for decreasing G-CSF during exercise.

In summary, therapeutic exercise improves both neutrophilic alveolar lung injury and skeletal muscle wasting in an animal model of ARDS. A short duration of acute exercise in ALI mice reduces neutrophil mobilization and recruitment to the lung and attenuates MuRF1mediated skeletal atrophy and the loss of specific force. Pharmacologic blockade of neutrophil mobilization and recruitment via G-CSF and other neutrophil chemokines are attractive targets to facilitate resolution of lung injury but may not attenuate the skeletal muscle wasting associated with ARDS. Early mobilization of patients with lung injury is a promising therapy to improve the outcomes of patients with ARDS. Additional studies are needed to further elucidate the mechanisms underlying its benefits, which may reveal new molecular targets for ARDS treatment.

\section{MATERIALS AND METHODS}

\section{Study design}

The aim of this study was to uncover mechanisms underlying the clinical benefits of early mobility therapy documented in various clinical studies. The study was performed using both a murine lung injury model and plasma from lung-injured humans undergoing early mobility therapy. The mouse studies were carried out by instillation of LPS into the lungs of mice. Subsets of mice underwent limb immobilization or performed therapeutic exercise after lung injury using a treadmill. Mice were randomly assigned to treatment groups, and where possible, the researchers were blinded to the treatment groups until statistical analysis. The predefined study endpoints included various aspects of muscle function and size, including maximal tetanic force, myofiber cross-sectional area, and quantification of musclewasting genes and proteins. Lung injury was quantified by alveolar cell counts and protein and histology scores. Systemic mediators of therapeutic exercise were quantified in mouse plasma with an inflammatory biomarker array. Quantification of lung and muscle injury parameters was performed with the following techniques: immunostaining, Western blotting, flow cytometry, ex vivo muscle stimulation, ELISA, and reverse transcription quantitative polymerase chain reaction (RT-qPCR).

The human mobility study was approved by the Wake Forest Human Subjects Committee and the Institutional Review Board and conducted from July 2007 to July 2009. Plasma was obtained from 93 patients randomized to receive early mobility (exercise) or usual care (no exercise). After performing our murine studies in 2013, we analyzed the banked plasma collected during the human study for quantification of G-CSF.

\section{ALI animal model}

All procedures were approved by the Institutional Animal Care and Use Committee of Wake Forest School of Medicine. Eight- to 12-weekold male wild-type C57BL/6 mice (The Jackson Laboratory) were anesthetized with an intraperitoneal injection of ketamine $(150 \mathrm{mg} / \mathrm{kg})$ and acetylpromazine $(13.5 \mathrm{mg} / \mathrm{kg})$, and the trachea was exposed. Escherichia coli LPS (O55:B5 L2880, lot 111M4035V, Sigma-Aldrich) (ARDS mice) at $3 \mu \mathrm{g} / \mathrm{g}$ mouse or an equivalent volume of sterile water (sham mice) was instilled intratracheally using a 20-gauge catheter as previously described (10).

\section{Hindlimb immobilization model}

A recently described hindlimb immobilization model using a surgical staple was applied as previously described (13). Sham and ALI mice underwent hindlimb immobilization at the time of i.t.LPS or i.t. $\mathrm{H}_{2} \mathrm{O}$ conditions by applying a surgical staple to the distal hindlimb in the normal flexion position. The contralateral hindlimb served as a control.

\section{Mouse treadmill}

Mice exercised on a six-lane mouse treadmill (Columbus Instruments) at $0^{\circ}$ incline at varying intensities and durations, using a graded protocol with durations ranging from $5 \mathrm{~min}$ once daily to $35 \mathrm{~min}$ twice daily. Exercise regimens began 24 hours after i.t.LPS instillation (day 1) and continued through day 2. Animals were sacrificed on day 3,12 hours after the final treadmill exercise. Speed was increased every $5 \mathrm{~min}$. Maximal tolerated speed or duration was defined as the lack of willingness to run on the treadmill for more than $2 \mathrm{~s}$ despite a $2-\mathrm{mA}$ shock. See table S1 for the dose and duration of each exercise group.

\section{In vivo G-CSFR inhibition}

A neutralizing antibody to the G-CSF receptor (G-CSFR/CD114 Ab, MAB6039, R\&D Systems) or isotype control (MAB0061, R\&D Systems) was administered to ALI mice at $0.5 \mu \mathrm{g}$ per mouse per day on days 1 and 2 after the administration of i.t.LPS (at day 0). The dose was based on a previous publication (27). For G-CSFR Ab prophylaxis experiments, G-CSFR Ab was administered at day -1 or 0 and continued daily through day 2 until harvest at day 3 .

\section{Other experiments}

Details of activity and lung injury measurements, muscle contractile and histology assessments, Western blotting, biomarker arrays, ELISAs, RT-qPCR, and the human mobility study can be found in Supplementary Materials and Methods.

\section{Statistics}

Data are presented as means \pm SEM. Data from each experiment were confirmed by two or more replicative experiments. The data presented are from representative or combined experiments where indicated. Differences between two groups were compared with the Student's $t$ test or the Mann-Whitney test for nonparametric data. Pairwise comparisons were performed using the Student's $t$ test. Survival was analyzed using the log-rank (Mantel-Cox) test. These data were analyzed with GraphPad Prism 6.0e (GraphPad Software). Inflammatory biomarker array data were analyzed using $t$ test statistics to get $P$ values for each protein, which are reported as uncorrected values without correction for multiplicity testing. The fold change for each protein was the ratio of the averaged intensities of the two groups. The human G-CSF data differences between groups over time were compared using the GEE implemented in R package GEEPACK (49), where the regression model included the interaction between treatment and time, and we used an unstructured working matrix. Human G-CSF data were normalized by adjusting all the data with the averaged value for each group at time zero. All raw data can be found in table S4.

\section{SUPPLEMENTARY MATERIALS}

www.sciencetranslationalmedicine.org/cgi/content/full/7/278/278ra32/DC1 Materials and Methods 
Fig. S1. Supplemental lung and muscle data from ALI and ALI + Ex mice. Fig. S2. Prophylactic blockade of the G-CSFR in ALI mice.

Table S1. Exercise protocols in lung-injured mice.

Table S2. Inflammatory biomarker array for ALI + Ex versus ALI mice.

Table S3. Patient characteristics at enrollment of randomized pilot study of early mobility in patients with ARF.

Table S4. Original data (provided as a separate Excel file).

References (50-52)

\section{REFERENCES AND NOTES}

1. P. E. Morris, A. Goad, C. Thompson, K. Taylor, B. Harry, L. Passmore, A. Ross, L. Anderson, S. Baker, M. Sanchez, L. Penley, A. Howard, L. Dixon, S. Leach, R. Small, R. D. Hite, E. Haponik, Early intensive care unit mobility therapy in the treatment of acute respiratory failure. Crit. Care Med. 36, 2238-2243 (2008).

2. C. Burtin, B. Clerckx, C. Robbeets, P. Ferdinande, D. Langer, T. Troosters, G. Hermans, M. Decramer R. Gosselink, Early exercise in critically ill patients enhances short-term functional recovery Crit. Care Med. 37, 2499-2505 (2009).

3. P. E. Morris, L. Griffin, M. Berry, C. Thompson, R. D. Hite, C. Winkelman, R. O. Hopkins, A. Ross, L. Dixon, S. Leach, E. Haponik, Receiving early mobility during an intensive care unit admission is a predictor of improved outcomes in acute respiratory failure. Am. J. Med. Sci. $\mathbf{3 4 1}$ 373-377 (2011).

4. D. M. Needham, R. Korupolu, J. M. Zanni, P. Pradhan, E. Colantuoni, J. B. Palmer, R. G. Brower, E. Fan, Early physical medicine and rehabilitation for patients with acute respiratory failure: A quality improvement project. Arch. Phys. Med. Rehabil. 91, 536-542 (2010).

5. W. D. Schweickert, M. C. Pohlman, A. S. Pohlman, C. Nigos, A. J. Pawlik, C. L. Esbrook, L. Spears, M. Miller, M. Franczyk, D. Deprizio, G. A. Schmidt, A. Bowman, R. Barr, K. E. McCallister, J. B. Hall, J. P. Kress, Early physical and occupational therapy in mechanically ventilated, critically ill patients: A randomised controlled trial. Lancet 373, 1874-1882 (2009).

6. P. Nydahl, A. P. Ruhl, G. Bartoszek, R. Dubb, S. Filipovic, H.-J. Flohr, A. Kaltwasser, H. Mende, O. Rothaug, D. Schuchhardt, N. Schwabbauer, D. M. Needham, Early mobilization of mechanically ventilated patients: A 1-day point-prevalence study in Germany. Crit. Care Med. 42, 1178-1186 (2014).

7. S. C. Berney, M. Harrold, S. A. Webb, I. Seppelt, S. Patman, P. J. Thomas, L. Denehy, Intensive care unit mobility practices in Australia and New Zealand: A point prevalence study. Crit. Care Resusc. 15, 260-265 (2013).

8. J. P. Kress, J. B. Hall, ICU-acquired weakness and recovery from critical illness. N. Engl. J. Med. 370, 1626-1635 (2014)

9. R. C. Langen, A. Haegens, J. H. J. Vernooy, E. F. M. Wouters, M. P. J. de Winther, H. Carlsen, C. Steele, S. E. Shoelson, A. M. W. J. Schols, NF-kB activation is required for the transition of pulmonary inflammation to muscle atrophy. Am. J. Respir. Cell Mol. Biol. 47, 288-297 (2012).

10. D. C. Files, F. R. D’Alessio, L. F. Johnston, P. Kesari, N. R. Aggarwal, B. T. Garibaldi, J. R. Mock, J. L. Simmers, A. DeGorordo, J. Murdoch, M. S. Willis, C. Patterson, C. G. Tankersley, M. L. Messi, C. Liu, O. Delbono, J. D. Furlow, S. C. Bodine, R. D. Cohn, L. S. King, M. T. Crow, A critical role for muscle ring finger-1 in acute lung injury-associated skeletal muscle wasting. Am. J. Respir. Crit. Care Med. 185, 825-834 (2012).

11. C. T. R. Gonçalves, C. G. R. Gonçalves, F. M. de Almeida, F. D. T. Q. dos Santos Lopes, A. C. C. dos Santos Durão, F. A. dos Santos, L. F. F. da Silva, T. Marcourakis, H. C. Castro-Faria-Neto, R. de Paula Vieira, M. Dolhnikoff, Protective effects of aerobic exercise on acute lung injury induced by LPS in mice. Crit. Care 16, R199 (2012).

12. B. T. Suratt, M. D. Eisner, C. S. Calfee, J. B. Allard, L. A. Whittaker, D. T. Engelken, J. M. Petty, T. Trimarchi, L. Gauthier, P. E. Parsons; NHLBI Acute Respiratory Distress Syndrome Network Plasma granulocyte colony-stimulating factor levels correlate with clinical outcomes in patients with acute lung injury. Crit. Care Med. 37, 1322-1328 (2009).

13. A. Z. Caron, G. Drouin, J. Desrosiers, F. Trensz, G. Grenier, A novel hindlimb immobilization procedure for studying skeletal muscle atrophy and recovery in mouse. J. Appl. Physiol. 106, 2049-2059 (2009).

14. V. Schefer, M. I. Talan, Oxygen consumption in adult and aged C57BL/6J mice during acute treadmill exercise of different intensity. Exp. Gerontol. 31, 387-392 (1996).

15. D. C. Files, K. Xiao, T. Zhang, C. Liu, J. Qian, W. Zhao, P. E. Morris, O. Delbono, X. Feng, The posterior cricoarytenoid muscle is spared from MuRF1-mediated muscle atrophy in mice with acute lung injury. PLOS One 9, e87587 (2014).

16. D. Cai, J. D. Frantz, N. E. Tawa Jr., P. A. Melendez, B.-C. Oh, H. G. W. Lidov, P.-O. Hasselgren, W. R. Frontera, J. Lee, D. J. Glass, S. E. Shoelson, IKK $/$ /NF-KB activation causes severe muscle wasting in mice. Cell 119, 285-298 (2004).

17. E. González, M. L. Messi, Z. Zheng, O. Delbono, Insulin-like growth factor-1 prevents agerelated decrease in specific force and intracellular $\mathrm{Ca}^{2+}$ in single intact muscle fibres from transgenic mice. J. Physiol. 552, 833-844 (2003).
18. K. P. Steinberg, J. A. Milberg, T. R. Martin, R. J. Maunder, B. A. Cockrill, L. D. Hudson, Evolution of bronchoalveolar cell populations in the adult respiratory distress syndrome. Am. J. Respir. Crit. Care Med. 150, 113-122 (1994).

19. F. J. Wiedermann, A. J. Mayr, N. C. Kaneider, D. Fuchs, N. J. Mutz, W. Schobersberger, Alveolar granulocyte colony-stimulating factor and $\alpha$-chemokines in relation to serum levels, pulmonary neutrophilia, and severity of lung injury in ARDS. Chest 125, 212-219 (2004).

20. J. L. Eyles, A. W. Roberts, D. Metcalf, I. P. Wicks, Granulocyte colony-stimulating factor and neutrophils-Forgotten mediators of inflammatory disease. Nat. Clin. Pract. Rheumatol. 2, 500-510 (2006).

21. E. Abraham, Neutrophils and acute lung injury. Crit. Care Med. 31, S195-S199 (2003).

22. E. Abraham, A. Carmody, R. Shenkar, J. Arcaroli, Neutrophils as early immunologic effectors in hemorrhage- or endotoxemia-induced acute lung injury. Am. J. Physiol. Lung Cell. Mol. Physiol. 279, L1137-L1145 (2000).

23. N. R. Aggarwal, F. R. D’Alessio, K. Tsushima, D. C. Files, M. Damarla, V. K. Sidhaye, M. M. Fraig, V. Y. Polotsky, L. S. King, Moderate oxygen augments lipopolysaccharide-induced lung injury in mice. Am. J. Physiol. Lung Cell. Mol. Physiol. 298, L371-L381 (2010).

24. R. P. Baughman, K. L. Gunther, M. C. Rashkin, D. A. Keeton, E. N. Pattishall, Changes in the inflammatory response of the lung during acute respiratory distress syndrome: Prognostic indicators. Am. J. Respir. Crit. Care Med. 154, 76-81 (1996).

25. M. A. Stark, Y. Huo, T. L. Burcin, M. A. Morris, T. S. Olson, K. Ley, Phagocytosis of apoptotic neutrophils regulates granulopoiesis via IL-23 and IL-17. Immunity 22, 285-294 (2005).

26. G. D. Demetri, J. D. Griffin, Granulocyte colony-stimulating factor and its receptor. Blood 78, 2791-2808 (1991).

27. M. Hara, S. Yuasa, K. Shimoji, T. Onizuka, N. Hayashiji, Y. Ohno, T. Arai, F. Hattori, R. Kaneda, K. Kimura, S. Makino, M. Sano, K. Fukuda, G-CSF influences mouse skeletal muscle development and regeneration by stimulating myoblast proliferation. J. Exp. Med. 208, 715-727 (2011).

28. F. Bussolino, M. Ziche, J. M. Wang, D. Alessi, L. Morbidelli, O. Cremona, A. Bosia, P. C. Marchisio, A. Mantovani, In vitro and in vivo activation of endothelial cells by colony-stimulating factors. J. Clin. Invest. 87, 986-995 (1991).

29. E. Azoulay, H. Attalah, K. Yang, S. Herigault, H. Jouault, C. Brun-Buisson, L. Brochard, A. Harf, B. Schlemmer, C. Delclaux, Exacerbation with granulocyte colony-stimulating factor of prior acute lung injury during neutropenia recovery in rats. Crit. Care Med. 31, 157-165 (2003).

30. C. Hierholzer, E. Kelly, V. Lyons, E. Roedling, P. Davies, T. R. Billiar, D. J. Tweardy, G-CSF instillation into rat lungs mediates neutrophil recruitment, pulmonary edema, and hypoxia. J. Leukoc. Biol. 63, 169-174 (1998).

31. H. Demuynck, P. Zachée, G. E. Verhoef, M. Schetz, G. Van den Berghe, P. Lauwers, M. A. Boogaerts, Risks of rhG-CSF treatment in drug-induced agranulocytosis. Ann. Hematol. 70, 143-147 (1995).

32. J. E. Rinaldo, H. Borovetz, Deterioration of oxygenation and abnormal lung microvascular permeability during resolution of leukopenia in patients with diffuse lung injury. Am. Rev. Respir. Dis. 131, 579-583 (1985).

33. R. K. Root, R. F. Lodato, W. Patrick, J. F. Cade, N. Fotheringham, S. Milwee, J.-L. Vincent, A. Torres, J. Rello, S. Nelson; Pneumonia Sepsis Study Group, Multicenter, double-blind, placebocontrolled study of the use of filgrastim in patients hospitalized with pneumonia and severe sepsis. Crit. Care Med. 31, 367-373 (2003).

34. S. Nelson, S. M. Belknap, R. W. Carlson, D. Dale, B. DeBoisblanc, S. Farkas, N. Fotheringham, H. Ho, T. Marrie, H. Movahhed, R. Root, J. Wilson, A randomized controlled trial of filgrastim as an adjunct to antibiotics for treatment of hospitalized patients with community-acquired pneumonia. J. Infect. Dis. 178, 1075-1080 (1998).

35. A. C. Cheng, D. P. Stephens, B. J. Currie, Granulocyte-colony stimulating factor (G-CSF) as an adjunct to antibiotics in the treatment of pneumonia in adults. Cochrane Database Syst. Rev., CD004400 (2007).

36. A. M. Greenbaum, D. C. Link, Mechanisms of G-CSF-mediated hematopoietic stem and progenitor mobilization. Leukemia 25, 211-217 (2010).

37. D. L. Rossi, S. D. Hurst, Y. Xu, W. Wang, S. Menon, R. L. Coffman, A. Zlotnik, Lungkine, a novel CXC chemokine, specifically expressed by lung bronchoepithelial cells. J. Immunol. 162, 5490-5497 (1999).

38. M. D. Giangola, W.-L. Yang, S. R. Rajayer, J. Nicastro, G. F. Coppa, P. Wang, Growth arrestspecific protein 6 attenuates neutrophil migration and acute lung injury in sepsis. Shock 40, 485-491 (2013).

39. X. O. Yang, S. H. Chang, H. Park, R. Nurieva, B. Shah, L. Acero, Y. H. Wang, K. S. Schluns, R. R. Broaddus, Z. Zhu, C. Dong, Regulation of inflammatory responses by IL-17F. J. Exp. Med. 205, 1063-1075 (2008)

40. A. Ichikawa, K. Kuba, M. Morita, S. Chida, H. Tezuka, H. Hara, T. Sasaki, T. Ohteki, V. M. Ranieri, C. C. dos Santos, Y. Kawaoka, S. Akira, A. D. Luster, B. Lu, J. M. Penninger, S. Uhlig, A. S. Slutsky, Y. Imai, CXCL10-CXCR3 enhances the development of neutrophil-mediated fulminant lung injury of viral and nonviral origin. Am. J. Respir. Crit. Care Med. 187, 65-77 (2013). 
41. C. Auvynet, S. Moreno, E. Melchy, I. Coronado-Martínez, J. L. Montiel, I. Aguilar-Delfin, Y. Rosenstein Galectin-1 promotes human neutrophil migration. Glycobiology 23, 32-42 (2012).

42. A. Pastva, K. Estell, T. R. Schoeb, T. P. Atkinson, L. M. Schwiebert, Aerobic exercise attenuates airway inflammatory responses in a mouse model of atopic asthma. J. Immunol. 172, 4520-4526 (2004).

43. R. P. Vieira, R. C. Claudino, A. C. S. Duarte, A. B. G. Santos, A. Perini, H. C. C. Faria Neto, T. Mauad, M. A. Martins, M. Dolhnikoff, C. R. F. Carvalho, Aerobic exercise decreases chronic allergic lung inflammation and airway remodeling in mice. Am. J. Respir. Crit. Care Med. 176, 871-877 (2007).

44. A. Moreira, L. Delgado, P. Moreira, T. Haahtela, Does exercise increase the risk of upper respiratory tract infections? Br. Med. Bull. 90, 111-131 (2009).

45. D. C. Nieman, Exercise, infection, and immunity. Int. J. Sports Med. 15 (Suppl. 3), S131-S141 (1994).

46. A. Pastva, K. Estell, T. R. Schoeb, L. M. Schwiebert, RU486 blocks the anti-inflammatory effects of exercise in a murine model of allergen-induced pulmonary inflammation. Brain Behav. Immun. 19, 413-422 (2005).

47. P. E. Hooijman, A. Beishuizen, M. C. de Waard, F. S. de Man, J. W. Vermeijden, P. Steenvoorde, R. A. Bouwman, W. Lommen, H. W. H. van Hees, L. M. A. Heunks, C. Dickhoff, D. L. van der Peet, A. R. J. Girbes, J. R. Jasper, F. I. Malik, G. J. M. Stienen, K. J. Hartemink, M. A. Paul, C. A. C. Ottenheijm, Diaphragm fiber strength is reduced in critically ill patients and restored by a troponin activator.Am. J. Respir. Crit. Care Med. 189, 863-865 (2014).

48. A. S. Moriscot, I. L. Baptista, J. Bogomolovas, C. Witt, S. Hirner, H. Granzier, S. Labeit, MuRF1 is a muscle fiber-type II associated factor and together with MuRF2 regulates type-II fiber trophicity and maintenance. J. Struct. Biol. 170, 344-353 (2010).

49. U. Halekoh, S. Højsgaard, J. Yan, The R package geepack for generalized estimating equations. J. Stat. Softw. 15, 1-11 (2006).

50. G. Y. Park, Y. G. Lee, E. Berdyshev, S. Nyenhuis, J. Du, P. Fu, I. A. Gorshkova, Y. Li, S. Chung, M. Karpurapu, J. Deng, R. Ranjan, L. Xiao, H. A. Jaffe, S. J. Corbridge, E. A. B. Kelly, N. N. Jarjour, J. Chun, G. D. Prestwich, E. Kaffe, I. Ninou, V. Aidinis, A. J. Morris, S. S. Smyth, S. J. Ackerman V. Natarajan, J. W. Christman, Autotaxin production of lysophosphatidic acid mediates allergic asthmatic inflammation. Am. J. Respir. Crit. Care Med. 188, 928-940 (2013).

51. T. Zhang, A. Birbrair, Z.-M. Wang, J. Taylor, M. L. Messi, O. Delbono, Troponin T nuclear localization and its role in aging skeletal muscle. Age 35, 353-370 (2013).
52. P.M. Siu, E. E. Pistilli, Z. Murlasits, S. E. Alway, Hindlimb unloading increases muscle content of cytosolic but not nuclear Id 2 and $\mathrm{p} 53$ proteins in young adult and aged rats. J. Appl. Physiol. 100, 907-916 (2006).

Acknowledgments: We thank P. Gann and R. Deaton at the University of Chicago Research Histology and Tissue Imaging Core for the digital lung imaging and analysis. D.C.F. thanks S. Kritchevsky and R. D. Hite for their mentorship and critical evaluation of the data. We thank the study participants, who made this work possible. Funding: These studies were supported by institutional funds from the Wake Forest School of Medicine, the Department of Medicine (D.C.F.), the Claude D. Pepper Older Americans Independence Center (P30-AG21332) (D.C.F., O.D., and P.E.M.), the Parker B. Francis Foundation (D.C.F.), the American Thoracic Society Foundation (D.C.F.), Wake Forest Health Sciences Translational Science Institute (M.S.), American Heart Association (FTF7280014) (N.R.A.), R00HL103973 (F.R.D.), NIH 1R01NR011186-01 (P.E.M.), NIH/National Institute on Aging R01AG13934 (O.D.), and R01AG15820 (O.D.). The funders had no role in study design, data collection and analysis, decision to publish, or preparation of the manuscript. Author contributions: Conceived and designed the experiments: D.C.F., O.D., N.R.A., and F.R.D. Performed the experiments: C.L., D.C.F., A.P., L.P., Z.-M.W., N.R.A., B.T.G., J.R.M., B.D.S., S.L., A.L.L., X.F., S.P., F.R.D., and M.S. Analyzed the data: D.C.F., J.C., A.P., N.R.A., and O.D. Contributed reagents/materials/analysis tools: D.C.F., M.S., L.S.K., O.D., J.C., R.R.Y., T.Z., and P.E.M. Wrote the manuscript: D.C.F. Provided edits to the manuscript: all authors. Competing interests: The authors declare that they have no competing interests. Data and materials availability: All data from this study have been made available to the journal.

Submitted 6 August 2014 Accepted 30 January 2015

Published 11 March 2015

10.1126/scitranslmed.3010283

Citation: D. C. Files, C. Liu, A. Pereyra, Z.-M. Wang, N. R. Aggarwal, F. R. D'Alessio, B. T. Garibaldi, J. R. Mock, B. D. Singer, X. Feng, R. R. Yammani, T. Zhang, A. L. Lee, S. Philpott, S. Lussier, L. Purcell, J. Chou, M. Seeds, L. S. King, P. E. Morris, O. Delbono, Therapeutic exercise attenuates neutrophilic lung injury and skeletal muscle wasting. Sci. Transl. Med. 7, 278ra32 (2015). 


\section{Therapeutic exercise attenuates neutrophilic lung injury and skeletal muscle wasting}

D. Clark Files et al.

Sci Transl Med 7, 278ra32 (2015);

DOI: 10.1126/scitranslmed.3010283

Editor's Summary

\section{Exercising away lung injury}

The benefits of exercise for healthy people are undisputed, but recent evidence suggests that exercise may have its place even among the sickest patients in the intensive care unit. New findings by Files et al. help explain these observations by using mouse models to find a mechanistic link between exercise and benefits in the acute respiratory distress syndrome, which they also confirm in human patients with respiratory failure who receive therapeutic exercise. The benefits of exercise in this setting include attenuation of muscle wasting as well as decreased lung inflammation. Although these improvements are both linked to exercise, they occur by different mechanisms, suggesting potential future approaches for more directed therapeutic intervention.

A complete electronic version of this article and other services, including high-resolution figures, can be found at:

http://stm.sciencemag.org/content/7/278/278ra32.full.html

Supplementary Material can be found in the online version of this article at: http://stm.sciencemag.org/content/suppl/2015/03/09/7.278.278ra32.DC1.html

Information about obtaining reprints of this article or about obtaining permission to reproduce this article in whole or in part can be found at:

http://www.sciencemag.org/about/permissions.dtl 